\title{
Stochastic Modeling of the Impact of Random Dopants on Hot-Carrier Degradation in n-FinFETs
}

\author{
Alexander Makarov, Ben Kaczer, Philippe Roussel, Adrian Chasin, Alexander Grill, Michiel Vandemaele, \\ Geert Hellings, Al-Moatasem El-Sayed, Tibor Grasser, Fellow, IEEE, Dimitri Linten, Stanislav Tyaginov
}

\begin{abstract}
Using the deterministic version of our hot-carrier degradation (HCD) model we perform a statistical analysis of the impact of random dopants (RDs) on HCD in n-FinFETs. For this, we use an ensemble of 200 transistors with different configurations of RDs. Our analysis shows that changes in the linear drain currents have broad distributions, thereby resulting in broad distributions of device lifetimes. While lifetimes are nearly normally distributed at high stress biases, under voltages close to the operating regime the distribution has a substantially different shape. This observation considerably complicates extrapolation from accelerated stress conditions, thereby suggesting that a comprehensive statistical treatment of the impact of RDs is required.
\end{abstract}

Index Terms-Hot-carrier degradation, random dopants, physical modeling, FinFETs, carrier transport, interface traps

\section{INTRODUCTION}

One of the most detrimental reliability concerns in ultrascaled field effect transistors (FETs) is hot-carrier degradation (HCD). Along these lines, HCD has been reported to be the main failure mode in the most recent FinFET node developed by Intel [1]. As a result, precise evaluation of device lifetime should include predictive modeling of HCD. Nevertheless, most HCD modeling approaches are empirical (i.e. rely on oversimplified expressions) and do not reveal the complex physical picture behind HCD. Herewith, they can only rely on backward extrapolation of device lifetime and fail when the physical mechanism driving HCD changes between stress and operating conditions. However, it is exactly this change that occurs in practice when the multiple-carrier (MC) mechanism of $\mathrm{Si}-\mathrm{H}$ bond rupture becomes dominant at lower stress voltages, in contrast to higher voltages where the single-carrier (SC) process governs HCD [2-4].

Another aspect which complicates modeling of reliability issues in modern FETs is the effect of random dopants (RDs). A nano-size transistor has just a handful of doping atoms with stochastically distributed locations [5]. Therefore, different samples from the same node inevitably have different configurations of RDs and the characteristics of pristine devices show substantial variability. Although nowadays some manufacturers (e.g. Intel $[6,7])$ use significantly reduced channel doping

This work was supported in part by the European Union's Horizon 2020 research and innovation programme under the Marie Sklodowska-Curie grant agreement No 794950 and by the Austrian Science Fund (FWF), grant No. P31204-N30.

A. Makarov, A.-M. El-Sayed and T. Grasser are with the Institute for Microelectronics, Technische Universität Wien, 1040 Vienna, Austria.

B. Kaczer, Ph. Roussel, A. Chasin, A. Grill, M. Vandemaele, G. Hellings, D. Linten, S. Tyaginov are with imec, Leuven 3001, Belgium. (e-mail: Stanislav.Tyaginov@imec.be)

to suppress RD induced fluctuations of device parameters, some other leading companies (such as IBM [8] and TSMC [9]) still employ FinFETs with "conventional" channel doping and therefore RD related variability still remains an issue.

Since HCD is driven by the interaction of hot and cold carriers $[4,10,11]$ and is thus determined by the carrier energy distribution function (DF), degradation proceeds with different rates in various samples. This issue has been addressed experimentally [12-15] and in simulation approaches [16-19] by different groups. However, all previous simulation approaches only provide a statistical description of HCD based on some phenomenological models which do not reveal the complex physical picture behind HCD which includes also the impact of RDs. The only model so far which attempts to capture RD induced variability of HCD has been published by Bottini et al. [20]. This model, however, does not consider the interplay between $\mathrm{SC}$ and $\mathrm{MC}$ mechanisms of $\mathrm{Si}-\mathrm{H}$ bond dissociation, which is of crucial importance for lifetime prediction.

To bridge this gap, we apply our physical HCD model which is based on a thorough carrier transport treatment [21,22] to cover the effect of RDs on HCD. Preliminary results of such a statistical description of HCD have already been presented in our recent paper [23]. However, in the current work the analysis will be performed over a wider set of stress conditions and more detailed explanations will be given.

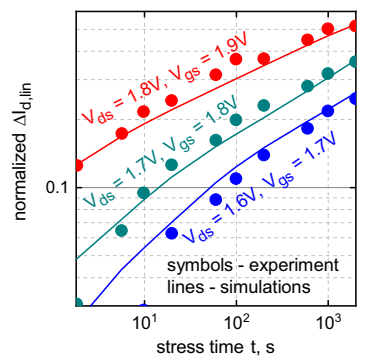

Fig. 1. The deterministic version of the model can accurately represent experimental $\Delta I_{\mathrm{d}, \text { lin }}(t)$ traces. The data are from our previous publication [25].

\section{The Modeling Framework}

Stochastic modeling of the effect of RDs on HCD will rely on the deterministic HCD model which has been shown to capture HCD in planar FETs [22], in high-voltage transistors [24], and in n-FinFETs [25]. This model (and the corresponding results) is referred to as "deterministic" because it employs continuous doping profiles (obtained by Sentaurus Process and labeled as "nominal") for computing carrier energy DFs 

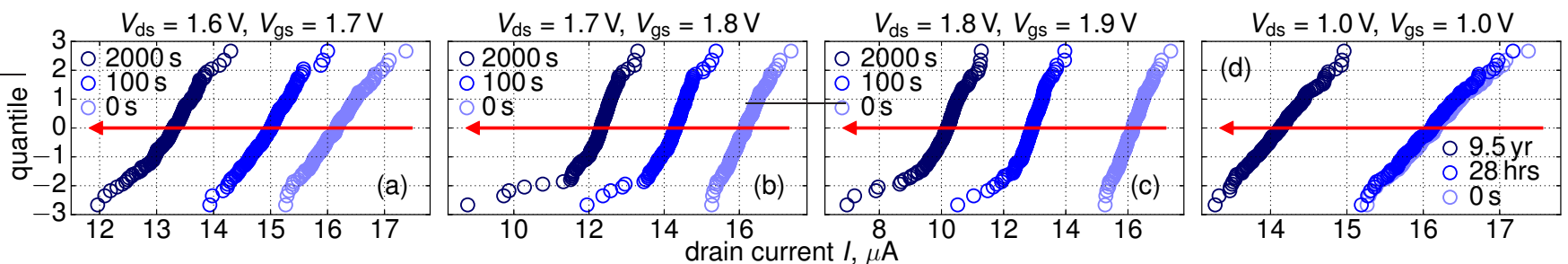

Fig. 3. The evolutions of the linear drain current distribution with time shown as probit plots for all four combinations of stress voltages. Data for $t=0 \mathrm{~s}$ correspond to pristine devices. Arrows correspond to increase of stress time.

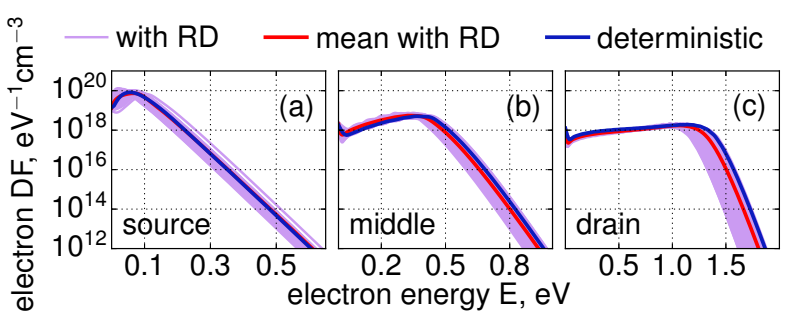

Fig. 4. Carrier DFs calculated for $200 \mathrm{RD}$ configurations $\left(V_{\mathrm{ds}}=1.8 \mathrm{~V}, V_{\mathrm{gs}}\right.$ $=1.9 \mathrm{~V}$ ) and shown at the source (a), in the middle of the channel (b), and at the drain (c). For comparison deterministic and mean DFs are also depicted.

and does not consider RD related variability. The calculated DFs are used to describe the interplay of the SC and MC mechanisms of $\mathrm{Si}-\mathrm{H}$ bond rupture and evaluate changes in device characteristics during stress (e.g. the normalized change of the linear drain current $\left.\Delta I_{\mathrm{d}, \text { lin }}\right)$ as a function of stress time $t$ (more details are given in [21,22]).

In this study, we use n-channel FinFETs with a channel length $L_{\mathrm{ch}}=28 \mathrm{~nm}$ (the corresponding gate length is $L_{\mathrm{g}}=$ $40 \mathrm{~nm})$ and a high- $k$ gate stack containing $\mathrm{SiO}_{2}$ and $\mathrm{HfO}_{2}$ layers with an equivalent oxide thickness of $1.2 \mathrm{~nm}$. The operating voltage is $V_{\mathrm{dd}}=0.9 \mathrm{~V}$. Our deterministic HCD model has been shown in our recent publication [25] to accurately capture $\Delta I_{\mathrm{d}, \text { lin }}(t)$ changes measured up to $2 \mathrm{ks}$ under three stress conditions $\left(I_{\mathrm{d}, \text { lin }}\right.$ corresponds to $V_{\mathrm{ds}}=0.05 \mathrm{~V}$ and $V_{\mathrm{gs}}$ $=0.9 \mathrm{~V}$, where $V_{\mathrm{ds}}$ and $V_{\mathrm{gs}}$ are drain and gate voltages, respectively): $V_{\mathrm{ds}}=1.6 \mathrm{~V}, V_{\mathrm{gs}}=1.7 \mathrm{~V} ; V_{\mathrm{ds}}=1.7 \mathrm{~V}, V_{\mathrm{gs}}=$ $1.8 \mathrm{~V}$; and $V_{\mathrm{ds}}=1.8 \mathrm{~V}, V_{\mathrm{gs}}=1.9 \mathrm{~V}$ (see Fig. 1 which presents data from [25]).

Based on the nominal architecture we generated a set of 200 devices with each of them having a unique configuration of RDs (see Fig. 2). To achieve this goal, for each mesh cell the continuous doping concentration is converted into the absolute number of doping atoms contained in that cell; this value is then used as the mean value for a Poisson random number generator which randomly draws the number of dopants in each of device realizations. For the entire set of pristine FinFETs we evaluated linear drain currents and binned them into probit plots (see Fig. 3) which show that currents are roughly normally distributed. These 200 configurations were used to calculate carrier energy DFs, interface state density $\left(N_{\mathrm{it}}\right)$ profiles, $\Delta I_{\mathrm{d}, \operatorname{lin}}(t)$ traces, and device lifetimes $(\tau)$. To obtain the DFs we solved the Boltzmann transport equation using the simulator ViennaSHE [26] which employs the spherical harmonics expansion method (for more details see [27,28]). Note that all calculations have also been performed for stress voltages $V_{\mathrm{ds}}=V_{\mathrm{gs}}=1.0 \mathrm{~V}$, which are close to the operating regime, in addition to the aforementioned high stress biases.

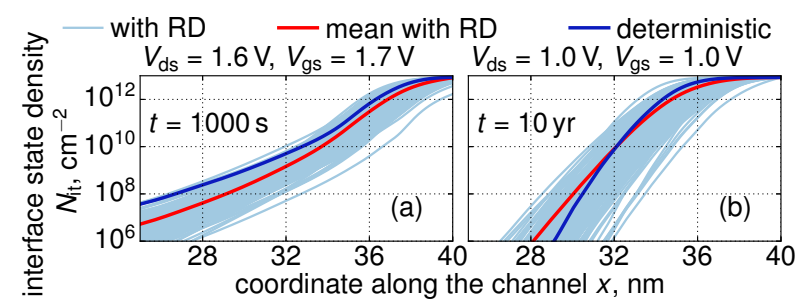

Fig. 5. $N_{\text {it }}$ profiles for all 200 devices computed for $V_{\mathrm{ds}}=1.6 \mathrm{~V}, V_{\mathrm{gs}}=$ $1.7 \mathrm{~V}$ at $t=1000 \mathrm{~s}$ (a) and $V_{\mathrm{ds}}=V_{\mathrm{gs}}=1.0 \mathrm{~V}$ at 10 years (b).

\section{RESUlTS AND DiscusSIONS}

The carrier DFs for the source, middle of the channel, and the drain obtained for all $200 \mathrm{RD}$ configurations (at $V_{\mathrm{ds}}=$ $1.8 \mathrm{~V}, V_{\mathrm{gs}}=1.9 \mathrm{~V}$ ) are summarized in Fig. 4. One can see that the nominal DFs have higher population numbers than the average over the ensemble. This trend is especially pronounced in the drain area (exactly where the damage is most severe) thereby suggesting that the deterministic model should result in overestimated HCD.

As for the $N_{\text {it }}$ profiles, they are summarized for two regimes with $V_{\mathrm{ds}}=1.6 \mathrm{~V}, V_{\mathrm{gs}}=1.7 \mathrm{~V}(t=1000 \mathrm{~s})$ and $V_{\mathrm{ds}}=V_{\mathrm{gs}}$ $=1.0 \mathrm{~V}(t \sim 10$ years) in Fig. 5. Note that for the sake of visibility the concentration $N_{\text {it }}$ is shown near the drain peak (the drain corresponds to the lateral coordinate of $x=40 \mathrm{~nm}$ ). From Fig. 5 we conclude that the deterministic model leads to $N_{\text {it }}$ concentrations higher than average $N_{\text {it }}$ values which is in accordance with the tendency visible in Fig. 4.

Fig. 3 shows evolutions of $I_{\mathrm{d}, \text { lin }}$ distributions with stress time. One can see that for short stress times and/or lower voltages currents are approximately normally distributed. For example, in the case of $V_{\mathrm{ds}}=1.6 \mathrm{~V}, V_{\mathrm{gs}}=1.7 \mathrm{~V}$ a deviation from the Gaussian distribution appears only after $\sim 2 \mathrm{ks}$, while for higher $V_{\mathrm{ds}}, V_{\mathrm{gs}}$ this non-normality is clearly visible already at $t=100 \mathrm{~s}$. As for $V_{\mathrm{ds}}=V_{\mathrm{gs}}=1.0 \mathrm{~V}$, the currents remain very closely normally distributed even after 10 years of stress.

The sets of $\Delta I_{\mathrm{d}, \operatorname{lin}}(t)$ degradation traces are depicted in Fig. 6 which clearly shows that in these scaled FinFETs HCD cannot be simply described by a single degradation curve but by an entire set of $\Delta I_{\mathrm{d}, \text { lin }}(t)$ dependences which can be very broad, thereby reflecting the impact of random dopants. Again, the mean $\Delta I_{\mathrm{d}, \text { lin }}$ values are lower than the nominal ones. Fig. 7 shows $\Delta I_{\mathrm{d}, \text { lin }}$ distributions evaluated for all $V_{\mathrm{ds}}, V_{\mathrm{gs}}$ combinations at three stress time steps. For all regimes, $\Delta I_{\mathrm{d}, \text { lin }}$ values are non-normally distributed (for $V_{\mathrm{ds}}=V_{\mathrm{gs}}=1.0 \mathrm{~V}$ this non-normality becomes pronounced only at long $t$ ). This peculiarity is probably related to the fact that samples with low time-zero currents $I_{\mathrm{d}, \text { lin }}(t=0)$ degrade slower and long $t$ can 

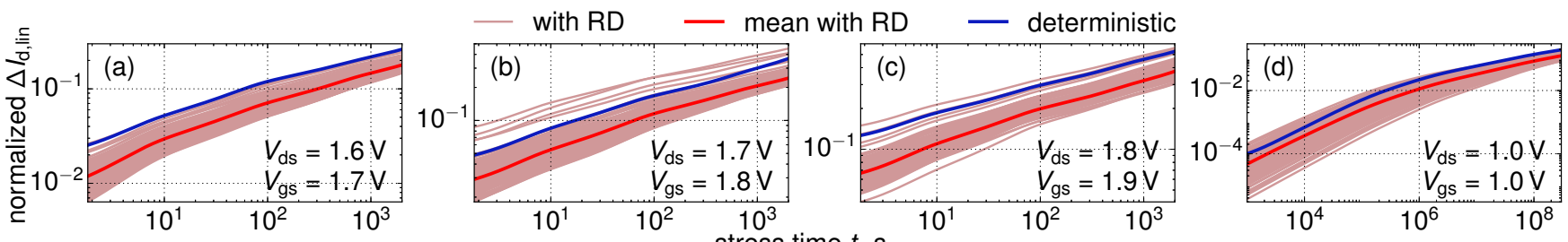

Fig. 6. Sets of $\Delta I_{\mathrm{d}, \text { lin }}(t)$ traces show very broad distributions for all combinations of stress voltages. One can see that average (over the device ensemble) $\Delta I_{\mathrm{d} \text {,lin }}$ values are significantly lower than those predicted with the deterministic version of our HCD model.
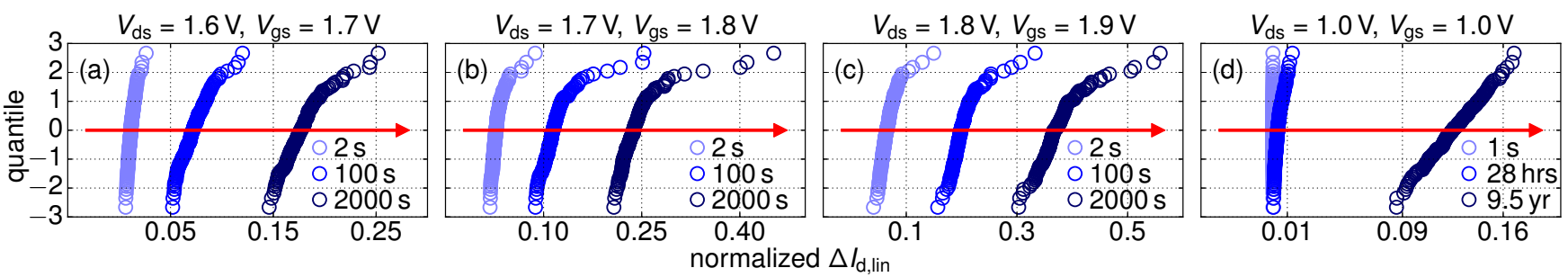

Fig. 7. Probit plots for $\Delta I_{\mathrm{d}, \text { lin }}$ evaluated at three different stress time steps.
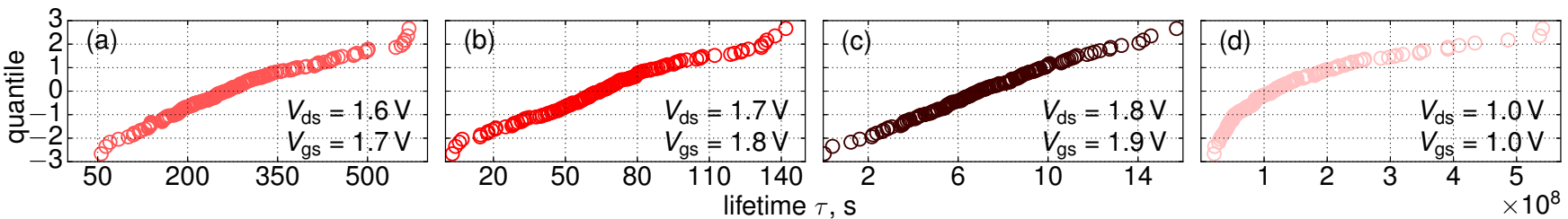

Fig. 8. Probit plots for device lifetimes extracted (based on the $\Delta I_{\mathrm{d}, \text { lin }}=10 \%$ criterion) from degradation traces data shown in Fig. 6 .
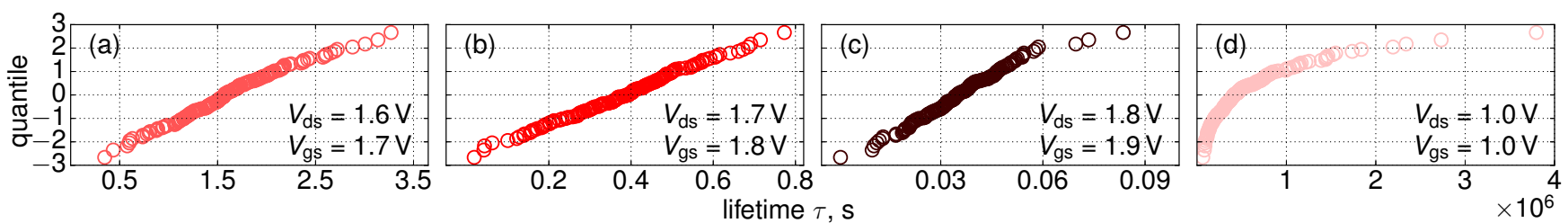

Fig. 9. Probit plots for device lifetimes extracted based on the $\Delta I_{\mathrm{d}, \mathrm{lin}}=1 \%$ criterion.

result in smaller $\Delta I_{\mathrm{d} \text {,lin }}$ values than with higher $I_{\mathrm{d}, \text { lin }}(t=0)$ values. However, investigation of this hypothesis requires more studies which will be published elsewhere. The data presented in Fig. 6 allowed us to extract device lifetimes $\tau$ (determined as the time at which $\Delta I_{\mathrm{d}}$, lin reaches $10 \%$ ) and bin them into distributions. Probit plots of these distributions (Fig. 8) show that for stress biases they are nearly normally distributed, while for $V_{\mathrm{ds}}=V_{\mathrm{gs}}=1.0 \mathrm{~V}$ the distribution is significantly nonnormal. For comparison, the $\tau$ distributions extracted based on the criterion of $\Delta I_{\mathrm{d}, \text { lin }}=1 \%$ (instead of $\Delta I_{\mathrm{d}, \text { lin }}=10 \%$ employed for Fig. 8) are summarized in Fig. 9. One can see that - although $\tau$ from Fig. 9 are shifted towards shorter values - the distributions from Figs. 8 and 9 have the same shapes at all combinations of $V_{\mathrm{ds}}, V_{\mathrm{gs}}$.

The trend that the deterministic HCD model results in HCD stronger than what is expected from the average over the FET ensemble is consistent with findings from $[29,30]$. Studies of the impact of RDs on the drain current, mobility, and threshold voltage in unstressed devices showed that the characteristics obtained for devices with continuous doping profiles significantly deviate from the mean values of these characteristics calculated taking the impact of RD into account. This effect is caused by the strong local perturbation of the device electrostatics by certain dopants. In the context of HCD, this perturbation depopulates the hot fraction of the carrier ensemble and thus weakens HCD.

\section{CONCLUSiOnS}

To analyze the effect of random dopants on hot-carrier degradation we generated a set of 200 n-channel FinFETs with different configurations of doping atoms. For each of these transistors we solved the Boltzmann transport equation and obtained carrier energy distribution functions. Then these DFs have been used to calculate ensembles of linear drain currents for each stress time step to extract degradation traces and device lifetimes. Our analysis has shown that $\Delta I_{\mathrm{d}, \operatorname{lin}}(t)$ traces have broad distributions and the deterministic version of the model (which considers only the continuous doping profiles without RD variations) results in $\Delta I_{\mathrm{d} \text {, lin }}$ values higher than average (over the device ensemble) current changes. As for linear drain currents, they are normally distributed in the pristine device and at short stress times, showing substantial deviations from the normal distribution at longer $t$ and/or higher voltages. Finally, lifetime distributions evaluated for stress conditions are close to normal, while for biases near the operating voltage the lifetime distribution has a completely different shape. This significantly complicates backward lifetime extrapolation and suggests that for a comprehensive HCD description which captures the effect of RD a full stochastic analysis is required. 


\section{REFERENCES}

[1] A. Rahman, J. Dacuna, P. Nayak, G. Leatherman, and S. Ramey, "Reliability Studies of a 10nm High-performance and Lowpower CMOS Technology Featuring 3rd Generation FinFET and 5th Generation HK/MG," in 2018 IEEE International Reliability Physics Symposium (IRPS), March 2018, pp. 6F.4-1-6F.4-6. DOI: 10.1109/IRPS.2018.8353648

[2] W. McMahon and K. Hess, "A Multi-Carrier Model for Interface Trap Generation," Journal of Computational Electronics, vol. 1, no. 3, pp. 395-398, Oct 2002. DOI: 10.1023/A:1020716111756

[3] A. Haggag, M. Lemanski, G. Anderson, P. Abramowitz, and M. Moosa, "Realistic Projections of Product Fmax Shift and Statistics due to HCI and NBTI," in 2007 IEEE International Reliability Physics Symposium (IRPS), April 2007, pp. 93-96. DOI: 10.1109/RELPHY.2007.369874

[4] A. Bravaix, C. Guerin, V. Huard, D. Roy, J. Roux, and E. Vincent, "HotCarrier Acceleration Factors for Low Power Management in DC-AC Stressed 40nm NMOS Node at High Temperature," in Proc. International Reliability Physics Symposium (IRPS), 2009, pp. 531-546. DOI: 10.1109/IRPS.2009.5173308

[5] A. Asenov, G. Slavcheva, A. R. Brown, J. H. Davies, and S. Saini, "Increase in the Random Dopant Induced Threshold Fluctuations and Lowering in sub-100 nm MOSFETs due to Quantum Effects: a 3D Density-gradient Simulation Study," IEEE Transactions on Electron Devices, vol. 48, no. 4, pp. 722-729, Apr 2001. DOI: 10.1109/16.915703

[6] S. Ramey, C. Prasad, and A. Rahman, "Technology Scaling Implications for BTI Reliability," Microelectronics Reliability, vol. 82, pp. 42-50, 2018. DOI: 10.1016/j.microrel.2018.01.004

[7] C. -Y. Su, M. Armstrong, L. Jiang, S. A. Kumar, C. D. Landon, S. Liu, I. Meric, K. W. Park, L. Paulson, K. Phoa, B. Sell, J. Standfest, K. B. Sutaria, J. Wan, D. Young, and S. Ramey, "Transistor Reliability Characterization and Modeling of the 22FFL FinFET Technology," in 2018 IEEE International Reliability Physics Symposium (IRPS), March 2018, pp. 6F.8-1-6F.8-7. DOI: 10.1109/IRPS.2018.8353652

[8] S. R. Stiffler, R. Ramachandran, W. K. Henson, N. D. Zamdmer K. McStay, G. La Rosa, K. M. Boyd, S. Lee, C. Ortolland, and P. C. Parries, "Process Technology for IBM 14-nm Processor Designs Featuring Silicon-On-Insulator FinFETs," IBM Journal of Research and Development, vol. 62, no. 2/3, pp. 11:1-11:7, March 2018. DOI 10.1147/JRD.2018.2800518

[9] J. H. Lee, Y. M. Shcu, C. C. Wu, Y. M. Liu, Y. C. Chou, and S. C. Chin, "An Electrical Failure Analysis (EFA) Flow to Quantitatively Identify Invisible Defect on Individual Transistor: Using the Characterization of Random Dopant Fluctuation (RDF) as an Example," in 2018 IEEE International Symposium on the Physical and Failure Analysis of Integrated Circuits (IPFA), July 2018, pp. 1-5. DOI: 10.1109/IPFA.2018.8452513

[10] K. Hess, A. Haggag, W. McMahon, K. Cheng, J. Lee, and J. Lyding, "The Physics of Determining Chip Reliability," IEEE Circuits and Devices Magazine, vol. 17, no. 3, pp. 33-38, May 2001. DOI: 10.1109/101.933789

[11] S. Tyaginov and T. Grasser, "Modeling of Hot-Carrier Degradation: Physics and Controversial Issues," in 2012 IEEE International Integrated Reliability Workshop Final Report, Oct 2012, pp. 206-215. DOI 10.1109/IIRW.2012.6468962

[12] P. Magnone, F. Crupi, N. Wils, H. P. Tuinhout, and C. Fiegna, "Characterization and Modeling of Hot Carrier-Induced Variability in Subthreshold Region," IEEE Transactions on Electron Devices, vol. 59, no. 8, pp. 2093-2099, Aug 2012. DOI: 10.1109/TED.2012.2200683

[13] E. R. Hsieh, S. S. Chung, C. H. Tsai, R. M. Huang, C. T. Tsai, and C. W. Liang, "New Observations on the Physical Mechanism of Vthvariation in Nanoscale CMOS Devices after Long Term Stress," in 2011 International Reliability Physics Symposium, April 2011, pp. XT.9.1XT.9.2. DOI: $10.1109 /$ IRPS.2011.5784610

[14] S. S. Chung, "The Process and Stress-Induced Variability Issues of Trigate CMOS Devices," in 2013 IEEE International Conference of Electron Devices and Solid-state Circuits, June 2013, pp. 1-2. DOI: 10.1109/EDSSC.2013.6628181

[15] B. Kaczer, J. Franco, M. Cho, T. Grasser, P. J. Roussel, S. Tyaginov, M. Bina, Y. Wimmer, L. M. Procel, L. Trojman, F. Crupi, G. Pitner, V. Putcha, P. Weckx, E. Bury, Z. Ji, A. D. Keersgieter, T. Chiarella, N. Horiguchi, G. Groeseneken, and A. Thean, "Origins and Implications of Increased Channel Hot Carrier Variability in nFinFETs," in 2015 IEEE International Reliability Physics Symposium, April 2015, pp. 3B.5.13B.5.6. DOI: 10.1109/IRPS.2015.7112706

[16] S. J. Bae, S. Kim, W. Kuo, and P. H. Kvam, "Statistical Models for Hot Electron Degradation in Nano-Scaled MOSFET Devices," IEEE
Transactions on Reliability, vol. 56, no. 3, pp. 392-400, Sep. 2007. DOI: 10.1109/TR.2007.903232

[17] Y. Li, S. Yu, J. Hwang, and F. Yang, "Discrete Dopant Fluctuations in 20-nm/15-nm-Gate Planar CMOS," IEEE Transactions on Electron Devices, vol. 55, no. 6, pp. 1449-1455, June 2008. DOI: 10.1109/TED.2008.921991

[18] L. Ma, X. Ji, Z. Chen, Y. Liao, F. Yan, Y. Song, and Q. Guo, "Physical Understanding of Hot Carrier Injection Variability in Deeply Scaled nMOSFETs," Japanese Journal of Applied Physics, vol. 53, no. 4S, p. 04EC15, jan 2014. DOI: 10.7567/JJAP.53.04EC15

[19] T. Hillebrand, N. Hellwege, N. Heidmann, S. Paul, and D. PetersDrolshagen, "Stochastic Analysis of Degradation and Variations in CMOS-Transistors," in ZuE 2015; 8. GMM/ITG/GI-Symposium Reliability by Design, Sep. 2015, pp. 1-8.

[20] R. Bottini, A. Ghetti, S. Vigano, M. G. Valentini, P. Murali, and C. Mouli, "Non-Poissonian Behavior of Hot Carrier Degradation Induced Variability in MOSFETs," in 2018 IEEE International Reliability Physics Symposium (IRPS), March 2018, pp. 6E.7-1-6E.7-6. DOI: 10.1109/IRPS.2018.8353645

[21] S. Tyaginov, M. Bina, J. Franco, D. Osintsev, O. Triebl, B. Kaczer and T. Grasser, "Physical Modeling of Hot-Carrier Degradation for Short- and Long-Channel MOSFETs," in Proc. International Reliability Physics Symposium (IRPS), 2014, pp. XT.16-1-16-8. DOI: 10.1109/IRPS.2014.6861193

[22] S. Tyaginov, M. Jech, J. Franco, P. Sharma, B. Kaczer, and T. Grasser, "Understanding and Modeling the Temperature Behavior of Hot-Carrier Degradation in SiON nMOSFETs," IEEE Electron Device Letters, vol. 37, no. 1, pp. 84-87, Jan 2016. DOI: 10.1109/LED.2015.2503920

[23] A. Makarov, B. Kaczer, P. Roussel, A. Chasin, A. Grill, M. Vandemaele, G. Hellings, E.-M. El-Sayed, T. Grasser, D. Linten, and S. Tyaginov, "Modeling the Effect of Random Dopants on Hot-Carrier Degradation in FinFETs," in Proc. International Reliability Physics Symposium (IRPS), 2019, pp. 6C.3.1-6C.3.6

[24] P. Sharma, S. Tyaginov, Y. Wimmer, F. Rudolf, K. Rupp, M. Bina, H. Enichlmair, J.-M. Park, R. Minixhofer, H. Ceric, and T. Grasser, "Modeling of Hot-Carrier Degradation in nLDMOS Devices: Different Approaches to the Solution of the Boltzmann Transport Equation," IEEE Transactions on Electron Devices, vol. 62, no. 6, pp. 1811-1818, 2015. DOI: 10.1109/TED.2015.2421282

[25] A. Makarov, S. E. Tyaginov, B. Kaczer, M. Jech, A. Chasin, A. Grill, G. Hellings, M. Vexler, D. Linten, and T. Grasser, "Hot-Carrier Degradation in FinFETs: Modeling, Peculiarities, and Impact of Device Topology," in Proc. International Electron Devices Meeting (IEDM), 2017, pp. 13.1.1-13.1.4. DOI: 10.1109/IEDM.2017.8268381

[26] http://viennashe.sourceforge.net/, 2014.

[27] K. Rupp, T. Grasser, and A. Jüngel, "On the Feasibility of Spherical Harmonics Expansions of the Boltzmann Transport Equation for Three-Dimensional Device Geometries," in 2011 International Electron Devices Meeting, Dec 2011, pp. 34.1.1-34.1.4. DOI: 10.1109/IEDM.2011.6131667

[28] M. Bina, K. Rupp, S. Tyaginov, O. Triebl, and T. Grasser, "Modeling of Hot Carrier Degradation Using a Spherical Harmonics Expansion of the Bipolar Boltzmann Transport Equation," in Proc. Inter national Electron Devices Meeting (IEDM), 2012, pp. 713-716. DOI 10.1109/IEDM.2012.6479138

[29] A. Asenov, R. Balasubramaniam, A. R. Brown, J. H. Davies, and S. Saini, "Random Telegraph Signal Amplitudes in sub 100 nm (Decanano) MOSFETs: a 3D 'Atomistic' Simulation Study,', in International Electron Devices Meeting 2000. Technical Digest. IEDM (Cat. No.00CH37138), Dec 2000, pp. 279-282. DOI: 10.1109/IEDM.2000.904311

[30] A. Asenov, F. Adamu-Lema, X. Wang, and S. M. Amoroso, "Problems with the Continuous Doping TCAD Simulations of Decananometer CMOS Transistors," IEEE Transactions on Electron Devices, vol. 61, no. 8, pp. 2745-2751, Aug 2014. DOI: 10.1109/TED.2014.2332034 